\section{Warum junge Patienten welche Therapie wählen}

Operieren, bestrahlen oder abwarten? Männer, bei denen schon sehr früh Prostatakrebs diagnostiziert wird, müssen diese schwierige Entscheidung treffen. Entsprechend viele Informationsquellen nutzen sie dafür.

M änner, bei denen vor dem 50. Lebensjahr Prostatakrebs diagnostiziert wird, stehen vor einem Dilemma: Aufgrund ihrer noch hohen Lebenserwartung benötigen sie eine kurative Therapie, aber therapiebedingte Folgen wie Impotenz oder Inkontinenz würden ihre Lebensqualität stärker als bei älteren Patienten beeinträchtigen. In einer US-amerikanischen Studie wurde untersucht, für welche Behandlungsoption sich Betroffene entscheiden und welche Informationen sie dafür nutzen. In dem Fragebogen, den 488 Männern ausfüllten, die zum Zeitpunkt ihrer Diagnose unter 50 Jahre alt waren, bezogen sich die Fragen u. a. auf Therapie, PSA-Wert, Informationsquellen, Entscheidungsfindung und Zufriedenheit mit der Entscheidung.

397 Patienten $(81,4 \%)$ wählten die radikale Prostatektomie (RP), 52 (10,7\%) eine Radiotherapie (RT) und 26 (5,3\%) Active Surveillance (AS). $\mathrm{Zu}$ den wich- tigsten Entscheidungshilfen zählten Urologe (96\%), Internet (58\%) und Partnerin (50\%). Über die Hälfte der Patienten konsultierte drei oder mehr Ärzte, bevor ein Entschluss gefasst wurde. Die häufigsten Gründe für eine RP waren die vermeintlich besten Heilungsaussichten und der Rat des Urologen. Patienten wählten die RT, um die invasivere RP und deren Folgen zu vermeiden. Gründe für AS waren Vermeidung von Operation und Nebenwirkungen sowie ein frühes Tumorstadium. Verheiratete Männer wählten signifikant häufiger RP oder RT, bei AS-Patienten waren hingegen die PSA-Werte signifikant niedriger. RP wurde in $99 \%$ der Fälle als Option diskutiert, Brachytherapie, RT und AS dagegen nur in 88 , 78 und $75 \%$ der Fälle.

Nicht verheiratete Männer taten sich schwerer, sich festzulegen, was laut den Autoren die Wichtigkeit eines stabilen sozialen Umfelds unterstreicht. Für die

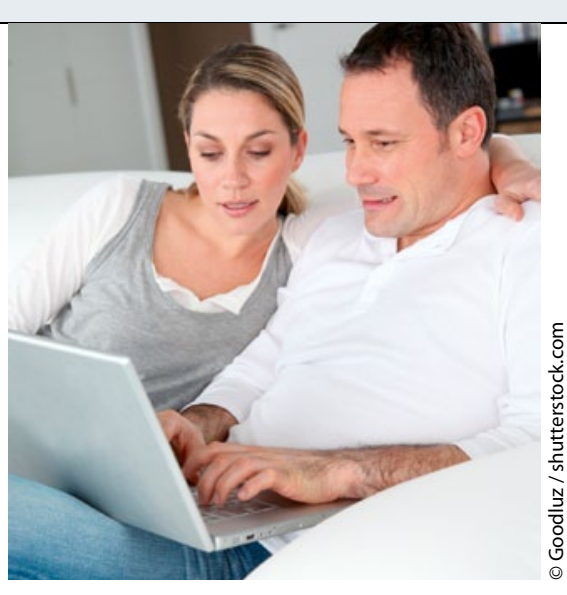

Bei der Therapieentscheidung können die Partnerin und das Internet helfen.

Männer in dieser Studie war der Erhalt der sexuellen Funktion wichtiger als die Kontinenz - in Studien mit Männern über 65 war das umgekehrt. $89 \%$ der Teilnehmer bereuten ihre Entscheidung nicht.

Fazit: Die Ergebnisse lassen sich sicher nicht zu $100 \%$ auf Deutschland übertragen, zeigen aber, wie mit Prostatakarzinom diagnostizierte junge Männer eine Therapie auswählen und welche Optionen ihre Ärzte empfehlen. Sebastian Lux

Sidana A et al. Treatment decision-making for localized prostate cancer: What younger men choose and why. Prostate. 2012;72(1):58-64.

\title{
PSA-Screening: keine Lösung in Sicht
}

\section{Seit 1991 läuft die European Randomized Study of Screening for Prostate Cancer (ERSPC). Jetzt gibt es neue Studiendaten, von denen man sich Antwort darauf erhofft, ob sich ein PSA-Screening rentiert.}

D ie Reduktion des relativen Risikos, an einem Prostatakarzinom zu sterben, bedeutete in der Studie: Um einen Tod aufgrund eines Prostatakarzinoms zu verhindern, mussten 1.055 Männer einem PSA-Test unterzogen und 37 Tumoren entdeckt werden - soweit der Stand der Dinge elf Jahre nach Studienbeginn.

Von den 182.160 Teilnehmern zwischen 50 und 74 Jahren waren 162.388 zwischen 50 und 69 Jahre alt. Das mediane Screeningintervall lag bei vier Jahren. Von durchschnittlich 2,27 Screeningtests pro Studienteilnehmer waren $16,6 \%$ positiv. Bei knapp 86\% der Männer mit positivem PSA-Test erfolgte eine Prostatabiopsie.

Auch die Ergebnisse der aktuellen Auswertung der ERSPC-Studie reichen nicht aus, um endgültig den Nutzen eines PSA-
Screenings zu belegen. Dazu müssten dessen Vor- und Nachteile sowie die Kosten genauer analysiert werden, so Fritz $\mathrm{H}$.

Das deckt sich mit dem aktuellen Entwurf der U.S. Preventive Services Task Force, in dem in den USA ein generelles PSA-Screening nicht mehr empfohlen wird. Genau das sei in Deutschland schon seit Jahren Politik, wie der Urologe Peter Albers aus Düsseldorf in einem Interview mit Springer Medizin sagt. Deshalb sei das Screening nicht verboten oder unsinnig - man müsse es nur intelligenter gestalten.

Fazit: Gegenüber der Situation vor zwei Jahren hat sich an den aktuellen Daten wenig geändert. In der Screeninggruppe sank das relative Risiko, an einem ProstaSchröder, Rotterdam, und Kollegen. takarzinom zu sterben, um $21 \%$ - nicht aber die Gesamtmortalität. Peter Leiner

Schröder FH et al. Prostate-cancer mortality at 11 years of follow-up. N Engl J Med. 2012; 366(11):981-90. - Editorial: Miller AB. New data on prostate-cancer mortality after PSA screening. Ibidem 1047-8.

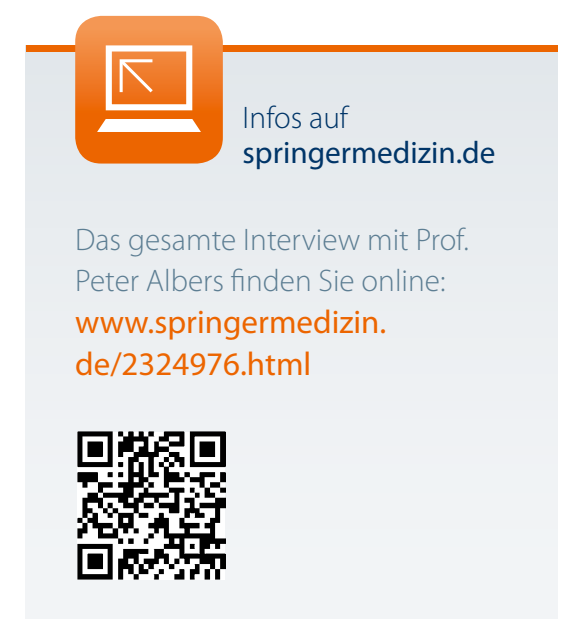

